Pacific Journal of Mathematics

FINDING A BOUNDARY FOR A HILBERT CUBE MANIFOLD 


\title{
FINDING A BOUNDARY FOR A HILBERT CUBE MANIFOLD BUNDLE
}

\author{
ScotT C. MetcalF
}

In this paper we develop an obstruction theory for the problem of determining whether a bundle, $E$, over a compact polyhedron, $B$, with non-compact Hilbert cube manifold fibers admits a boundary in the sense that there exists a compact bundle $\bar{E}$ over $B$ with $Q$-manifold fibers and a sliced $Z$-set, $A \subset \bar{E}$,such that $\bar{E}=A \cup E$. Included in the work is a new result on fibered weak proper homotopy equivalences, a theorem on proper liftings of homotopies, and the development of a sliced shape theory whose equivalences are shown to classify our boundaries through a tie to $Q$-manifold theory via a sliced version of Chapman's Complement Theorem.

1. Introduction. Browder, Levine, and Livesay, [3], and Siebenmann, [26], studied the question of putting a boundary on a finite dimensional manifold. Adopting a somewhat more general, $Z$-set notion of a boundary, Chapman and Siebenmann successfully obtained an obstruction theory for the problem of determining which $Q$-manifolds admit boundaries in [14]. Interest has since been expressed in the boundary problem in a (locally trivial) bundle setting (see the problem section in the back of [8] and, more recently, problem $(Q M 10)$ in [20].) The purpose of this paper is to investigate the question of when a bundle with non-compact $Q$-manifold fibers can be compactified in such a way that the resulting manifold has a bundle structure extending the original bundle structure. In another variation on the boundary problem Chapman, [9], has developed machinery for deciding if a $Q$-manifold admits a controlled boundary. The boundary is controlled in the sense that one is given an arbitrary map from the non-compact manifold to a parameter space and desires to find a compactification along with an extension of the given map. The controlled boundary problem is evidently more general than the question studied in this paper and it would be interesting to investigate the relationship between the results of the two theories.

We begin with the introduction of some terminology and notation. All spaces except function spaces will be locally compact, separable metric spaces. A (locally trivial) bundle, $M \stackrel{p}{\rightarrow} B$, will be called a $Q$-manifold bundle if its fiber, $F$, is a $Q$-manifold, i.e. a manifold modeled on the 
Hilbert cube, $Q$. The usual procedure of treating components of $B$ separately and, hence, assuming $B$ is connected allows us to talk about the fiber of $M \stackrel{p}{\rightarrow} B$. The base space, $B$, will be assumed to be a compact polyhedron and the bundle called a non-compact $Q$-manifold bundle if its fiber, $F^{Q}$, is noncompact. In this setting, a sliced Z-set of $M \stackrel{p}{\rightarrow} B$ is a closed set $A \subset M$ with the property that there exist fiber preserving (f.p.) maps $M \rightarrow M-A$ arbitrarily close to the identity. With this notation we consider when, given a non-compact $Q$-manifold bundle $M \stackrel{p}{\rightarrow} B$, we can find a compact $Q$-manifold bundle $\tilde{p}: \tilde{M} \rightarrow B$ such that $\tilde{M}=M \cup A$ where $A$ is a sliced $Z$-set of $\tilde{p}: \tilde{M} \rightarrow B$ and $\tilde{p} \mid M=p$.

Clearly $p: M \rightarrow B$ admitting a bundle compactification implies that both its fiber, $F$, and $M$ itself admit boundaries in the sense of ChapmanSiebenmann. In fact, for $B$ equal a point the bundle boundary problem reduces to the problem considered by Chapman and Siebenmann. Just as Chapman and Siebenmann observed basic necessary conditions for a $Q$-manifold to admit a boundary, i.e. tameness at $\infty$ and finiteness of type, we can easily recognize the necessity of fibered versions of these conditions. Specifically we say $p: M \rightarrow B$ is slicewise tame at $\infty$ if for every compactum $C \subset M$ there exists a compactum $C^{\prime}$ with $C \subset C^{\prime} \subset M$ such that $M-C^{\prime} \hookrightarrow M-C$ factors slicewise through a finite complex up to fibered homotopy; i.e. there exists a finite polyhedron $K$, maps $\alpha$ and $\beta$, and a f.p. homotopy $H: i \simeq \beta \circ \alpha$ making the following diagram commute (up to fibered homotopy)

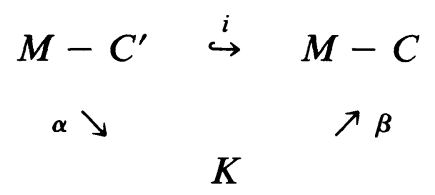

In a similar spirit, $p: M \rightarrow B$ is said to have sliced finite type if it is fibered homotopy equivalent to a bundle over $B$ with compact $Q$-manifold fibers. In light of the triangulation of compact $Q$-manifold bundles (see Chapman and Ferry [12] for a proof using classifying spaces or [13] for a more geometric proof) we could equivalently demand that the given bundle be homotopy equivalent to a bundle with fiber a finite polyhedron.

Verifying that these necessary conditions which we have isolated hold in a particular bundle is then the first step in determining whether it admits a boundary. In checking for sliced finite type we encounter what Hatcher refers to as the fibered obstruction to finiteness in problem 6.1 of [22]. This problem can be formulated as a lifting problem which we briefly describe. Let $X$ be a finite polyhedron which is homotopy equivalent to 
the fiber of the bundle $p: M \rightarrow B$. Regarding $p: M \rightarrow B$ as a Hurewicz fibration $p$ is classified by a map of the base $B$ into the classifying space of self-homotopy equivalences of $X, B \rightarrow B G(X)$. Write $B(X)$ for a universal space for PL fibrations of finite polyhedra with fibers homotopy equivalent to $X$. The lifting problem is that of completing the commutative diagram

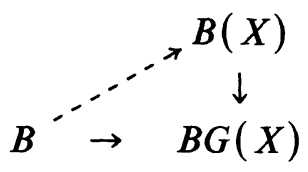

where $B(X) \rightarrow B G(X)$ is the natural forgetful map. Generalizing the simple homotopy equivalences of [16] is the notion of a simple map, i.e. a PL map of finite polyhedra with contractible point inverses. Using simple maps as morphisms yields a category $S(X)$ whose objects are finite polyhedra containing $X$ as a deformation retract. The obstructions to sliced finite type then come from $\Pi_{*} B S(X)(B S(X)$ being the classifying space of $S(X))$ since $B S(X)$ can be shown to be the homotopy fiber of $B(X) \rightarrow B G(X)$, see [21]. The obstruction then lies in the realm of Hatcher's higher simple-homotopy theory, the name justified by the observation that $\Pi_{0} B S(X) \approx \mathrm{Wh}\left(\Pi_{1} X\right)$.

The main result of this paper identifies a second obstruction for a bundle assumed to satisfy the necessary conditions above.

THEOREM 1. Homotopy Boundary Theorem. A non-compact Q-manifold bundle which has sliced finite type and is slicewise tame at $\infty$ is proper fiber homotopy equivalent to one which admits a bundle compactification.

Chapman and Siebenmann were able to characterize $Q$-manifolds which admit boundaries as those homeomorphic to a product of $Q$ times an infinite mapping cylinder, $\operatorname{Map}(\sigma)$, where $\sigma$ is an inverse sequence of compact polyhedra arising from the tameness at $\infty$ condition imposed on the manifold. Requiring a bundle for our target we use a compact $Q$-manifold bundle which we get from sliced finite type and remove a sliced $Z$-set copy of $\lim \sigma$ from it. Chapman and Siebenmann were able to identify what they called the total obstruction (to the unfibered boundary problem) as lying in the quotient of the group of simple-homotopy types on $M$ by the Whitehead group of $\Pi_{1} M$. In analogy it seems likely that proper definitions of a fiber simple-homotopy theory will locate the obstruction to a fibered boundary as the image of the (fibered) torsion of the proper fiber homotopy equivalence from Theorem 1 in the quotient of 
the group of all fiber simple homotopy types on $M \rightarrow B$ by an appropriate fibered Whitehead group. The following theorem assures us that the obstruction is well-defined.

THEOREM 2. If $f: N \rightarrow N^{\prime}$ is a proper fiber homotopy equivalence between non-compact $Q$-manifold bundles which admit bundle compactifications then $f$ is proper fiber homotopic to a fiber-preserving homeomorphism near $\infty$.

As an application we prove the following.

COROllary 3. If $p: M \rightarrow S^{1}$ is a non-compact $Q$-manifold bundle which is sliced tame at $\infty$ and its fiber, $F$, is simply connected and simply connected at $\infty$ then $M$ admits a bundle compactification.

A notable ingredient of the proof of the corollary is the calculation that $\Pi_{0} \mathscr{C}(F)=0$ where $\mathscr{C}(F)$ is the group of concordances of $F$ (see $\S 8$ for a precise definition), a result made possible by Chapman's exact sequences of [7]. For bundles with higher dimensional bases one would need assumptions of higher connectivity on the fiber as suggested by the work of Burghelea, Lashof and Rothenberg, see for example Theorem B' on page 36 of [4].

We introduce a sliced shape theory in this paper and prove a sliced version of Chapman's Complement Theorem which is of fundamental importance in the proofs of the theorems above. In addition we prove the following

Boundary Classification Theorem. Let $A \stackrel{p}{\rightarrow} B$ be a boundary for the noncompact $Q$-manifold bundle $E \stackrel{p}{\rightarrow} B$ and $A^{\prime}$ be a compact metric space. $A^{\prime} \stackrel{p^{\prime}}{\rightarrow} B$ is a boundary for $E \stackrel{p}{\rightarrow} B$ if and only if $A$ and $A^{\prime}$ have the same sliced shape.

As to the organization of this paper we state fibered $Q$-manifold preliminaries in $\$ 2$. Section 3 contains sliced shape theory definitions and results including our sliced shape complement theorem and the proof of the Boundary Classification Theorem. In $\$ 4$ we treat weak proper homotopy equivalences indicating a fibered version of a result of Edwards and Hastings [18]. The preliminary groundwork is completed with the proof 
that small homotopies have proper lifts and thus, using Dold's trick ([17]), small homotopy equivalences between bundles yield proper fiber homotopy equivalences in $\$ 5$.

Section 6 contains the proof of Theorem 1 while $\$ 7$ has that of Theorem 2. Finally a brief discussion of calculations using these results along with the proof of Corollary 3 may be found in $\$ 8$.

2. Fibered $Q$-manifold preliminaries. The following definitions and notation will be used throughout this work. Letting $I=[0,1]$, we say that a homotopy $H: X \times I \rightarrow Y$ is an $\alpha$-homotopy or is limited by $\alpha$, a cover of $Y$, if $H(\{x\} \times I)$ is contained in some element of $\alpha$ for any $x \in X$. We write $f \approx_{\text {f.p. }} g$ to indicate that maps $f$ and $g$ are homotopic through a f.p. homotopy and $f \sim_{\alpha} g$ if $f, g: X \rightarrow Y$ are $\alpha$-close, i.e. for any $x \in X$ there exists an element $0 \in \alpha$ such that $f(x)$ and $g(x)$ both lie in 0 .

A homeomorphism near $\infty$ is a map, i.e. a continuous function, $f$ : $X \rightarrow Y$ such that for any compactum $C \subset X$ there exists a compactum $C^{\prime}$ containing $C$ with $\left.f\right|_{X \sim C^{\prime}}: X-C^{\prime} \rightarrow Y-f\left(C^{\prime}\right)$ a homeomorphism. A map $f: X \rightarrow Y$ between absolute neighborhood retracts is cell-like (CE) if it is a proper surjection and for every $y \in Y$ and every open set $U$ with $f^{-1}(y) \subset U$ there exists an open set $V \subset U$ which contains $f^{-1}(y)$ and deforms to a point in $U$. Basic definitions of the proper and weak proper categories are collected in $\$ 4$ while $\$ 5$ contains fibered shape definitions.

A $Q$-manifold is a separable metric manifold locally modeled on the Hilbert cube. The basic theory can be found in [8] where it is readily apparent that $Z$-sets play the key role. The main tools of $Q$-manifold theory, stability, mapping replacement and $Z$-set unknotting have been observed to hold in product ([15], [19] and [10]), bundle ([29]), and submersive ([24]) settings. Fibered versions of Miller's theorem and CE approximation as well as characterization and triangulation of $Q$-manifold bundles have also been proven. Below are versions of the $Q$-manifold tools we will use frequently in what follows. Statements are taken from [29] and [24].

Fibered Mapping Replacement. Let $p: M \rightarrow B$ be a $Q$-manifold bundle and $f: A \rightarrow M$ a proper map. Suppose that $A_{0}$ is a compact subset of $A$ and $\left.f\right|_{A_{0}}$ is a sliced $Z$-embedding. Then for any open cover $\alpha$ of $M$ there exists a proper map $\hat{f}: A \rightarrow M$ such that

(i) $p \hat{f}=p f$

(ii) $\hat{f}$ is a sliced $Z$-embedding

(iii) $\hat{f}=f$ on $A_{0}$ and

(iv) $\hat{f}$ is $\alpha$-close to $f$. 
Fibered Z-Set Unknotting. Let $p: M \rightarrow B$ be a $Q$-manifold bundle, $A$ a compactum and $F: A \times I \rightarrow M$ a map such that $F_{0}$ and $F_{1}$ are sliced $Z$-embeddings. If, additionally, $p F_{t}(a)=p F_{0}(a)$ for all $a \in A, F(A \times I)$ $\subset U$ an open set of $M$, and $F$ is limited by an open cover $\alpha$ of $U$ then there exists an isotopy $H: M \times I \rightarrow M$ satisfying

(i) $H_{0}=$ id

(ii) $H_{t} \mid M \backslash U=$ id

(iii) $H \mid U \times I$ is an $\alpha$-isotopy

(iv) $p H_{t}=p$ and

(v) $H_{1} F_{0}=F_{1}$.

It should be noted that the result holds for $A$ merely locally compact if $F$ is a proper map.

Fibered Stability. For any $Q$-manifold bundle $p: M \rightarrow B$ and any open cover $\alpha$ of $M$ there exists a f.p. homeomorphism $h: M \times Q \rightarrow M$ which is $\alpha$-close to projection.

Fibered Collaring. If $p: M \rightarrow B$ is a $Q$-manifold bundle and, for $M_{1}$ a sliced $Z$-set of $M,\left.p\right|_{M_{1}}: M_{1} \rightarrow B$ is also a $Q$-manifold bundle then $M_{1}$ has a fibered collar in $M$, i.e. there exists an open embedding $M \times[0,1) \hookrightarrow M$ with $M_{1} \times\{0\}=M_{1}$ and $p(m, t)=p(m, 0)$ for all $t \in[0,1)$ and all $m \in M_{1}$.

The following are unfibered results from $Q$-manifold theory which we will use. As noted above, the first is true in a bundle setting, a fact easily proved by piecing unfibered $\mathrm{CE}$ approximations on fibers together by local contractibility over a fine triangulation of $B$, but we shall not need the fibered result.

CE APPRoximation Theorem. CE maps between $Q$-manifolds can be approximated arbitrarily close by homeomorphisms.

Classification Theorem. Suppose that $f: X \rightarrow Y$ is a proper map between polyhedra. $f$ is a simple homotopy equivalence if and only if $f \times \mathrm{id}_{Q}$ : $X \times Q \rightarrow Y \times Q$ is proper homotopic to a homeomorphism.

Standard references for simple homotopy theory are [16] and, for non-compact spaces, [27]. 
3. Shape theory in the sliced category. Chapman established the connection between $Q$-manifolds and shape theory in [5] (see also [8]). In this section we give definitions for a parameterized shape theory, limiting our discussion to the case in which the parameterization is actually a bundle map. We present both the traditional approach of Borsuk using fundamental sequences, [2], and the ANR-system approach of Mardešić and Segal as both are useful and they are equivalent. The relevance of sliced shapes to $Q$-manifold bundles is established in a sliced version of Chapman's Complement Theorem which is important in the proofs of both of our main theorems. In the Homotopy Boundary Theorem the application takes a form which we isolate as a corollary. Throughout we assume that $p: E \rightarrow B$ is a $Q$-manifold bundle.

Let $A$ and $A^{\prime}$ be compact subsets of $E$. A relative shape map from $A$ to $A^{\prime}$ sliced over $B$ consists of an open set $G$ of $E$ containing $A$ and a sequence of f.p. maps $f_{k}$ : $G \rightarrow E$ satisfying (i) $f_{k}$ is fiber homotopic to the inclusion $G \hookrightarrow E$ for all $k$, and (ii) for every neighborhood $V$ of $A^{\prime}$ there exists an open neighborhood $U$ of $A$ in $G$ and an integer $N>0$ such that $k, l \geq N$ implies $\left.\left.f_{k}\right|_{U} \simeq_{\text {f.p. }} f_{l}\right|_{U}$ (in $V$ ). We shall write $\underline{f}=\left\{f_{n}, A, A^{\prime}, G\right\}$ : $A \rightarrow A^{\prime}$ for such a relative sliced shape map.

Given two relative sliced shape maps $\underline{f}=\left\{f_{n}, A, A^{\prime}, G\right\}$ and $\underline{g}=$ $\left\{g_{n}, A, A^{\prime}, G\right\}$ we say $\underline{f}$ is homotopic to $\underline{g}$ (written $\underline{f} \simeq \underline{g}$ ) if for every neighborhood $V$ of $A^{\prime}$ there exists a neighborhood $U$ of $A$ and an integer $N>0$ such that $k \geq N$ implies $\left.\left.f_{n}\right|_{U} \simeq_{\text {f.p. }} g_{n}\right|_{U}$ (in $V$ ). The identity shape map is $\operatorname{id}_{A}=\left\{\operatorname{id}_{G}, A, A, G\right\}$. Suppose that $\underline{f}=\left\{f_{n}, A, A^{\prime}, G\right\}$ and $\underline{g}=$ $\left\{g_{n}, A^{\prime}, \bar{A}^{\prime \prime}, H\right\}$ are relative sliced shape maps between compacta in $E$. Their composition is defined by $g f=\left\{g_{n} f_{n}, A, A^{\prime \prime}, G^{\prime}\right\}$ where $G^{\prime}$ is a suitably restricted neighborhood of $A$. Clearly this composition is a relative sliced shape map from $A$ to $A^{\prime \prime}$.

A relative sliced shape map $\underline{f}: A \rightarrow A^{\prime}$ is a relative sliced shape equivalence if there exists a relative sliced shape map $\underline{g}: A^{\prime} \rightarrow A$ such that $\underline{f g} \simeq \mathrm{id}_{A^{\prime}}$ and $\underline{g f} \simeq \mathrm{id}_{A} . A$ and $A^{\prime}$ then are said to have the same relative sliced shape, denoted by $\operatorname{Sh}_{B}^{E}(A)=\operatorname{Sh}_{B}^{E}\left(A^{\prime}\right)$.

The definition of the relative sliced shape of a compacta $A \subset E$ coincides with the standard, unsliced definition of relative shape if one takes $B=*$, a point. For the passage to the absolute case, let a compactum $A$ and a map $p: A \rightarrow B$ be given. Consider the trivial bundle $\operatorname{proj}_{B}: B \times Q \rightarrow B$. Regard $A$ as a subset of $B \times Q$ by choosing a sliced $Z$-embedding near the map $j: A \rightarrow B \times Q$ where $j(a)=(p(a), \mathbf{0}), \mathbf{0} \in Q$. Then the absolute sliced shape of $A, \mathrm{Sh}_{B}(A)$, is defined by $\operatorname{Sh}_{B}(A)=$ $\mathrm{Sh}_{B}^{B \times Q}(A)$. 
The approach to shape given above is patterned after Borsuk's definition and is useful in the proof of our

Sliced Shape Complement Theorem. Let $A$ and $A^{\prime}$ be compact, sliced $Z$-sets of $E . \operatorname{Sh}_{B}^{E}(A)=\operatorname{Sh}_{B}^{E}\left(A^{\prime}\right)$ if and only if there exists a f.p. homeomorphism $h: E \backslash A \rightarrow E \backslash A^{\prime}$ with $h \simeq_{\text {f.p. }} \mathrm{id}_{E}$. (Actually the homotopy is between the identity and the composition $E \rightarrow E \backslash A \stackrel{h}{\rightarrow} E \backslash A^{\prime} \hookrightarrow E$, the first map being a fibered push off of $A$ by a map which is close to the identity.)

For the direction which asserts that a f.p. homeomorphism between complements implies that the compacta have the same shape we use the following lemma which gives an explicit f.p. homotopy pushing the total space of a $Q$-manifold bundle off of a sliced $Z$-set instantaneously.

LemMA. For any sliced Z-set $A$ of $p: E \rightarrow B$ there exists a homotopy $F$ : $E \times I \rightarrow E$ satisfying

(i) $F_{t}(E) \subset E \backslash A$ for all $t>0$,

(ii) $F_{0}=$ id,

(iii) $F$ is f.p., and

(iv) for every open neighborhood $U$ of $A$ there exists $t_{0} \in(0,1)$ such that $F_{t}(U) \subset U$ for $0 \leq t \leq t_{0}$.

Shape maps which establish the shape equivalence of $A$ and $A^{\prime}$ are then given by $f_{n}=h F_{1 / n}$ and $g_{n}=h^{-1} G_{1 / n}$ where $G_{t}: E \rightarrow E$ comes from the lemma with $A$ replaced by $A^{\prime}$.

For the other direction one uses the shape equivalence from $A$ to $A^{\prime}$ to construct a sequence of homeomorphisms $h_{i}$ of $E$ onto itself such that the composition $h_{n} h_{n-1} \cdots h_{2} h_{1}$ pushes $A$ closer and closer to $A^{\prime}$ as $n$ increases. The construction makes repeated used of fibered mapping replacement and $Z$-set unknotting and is accomplished in such a way that points in $E \backslash A$ are moved by only finitely many of the $h_{i}$. This last condition makes the map $h=\left.\lim _{n \rightarrow \infty} h_{n} \cdots h_{2} h_{1}\right|_{E \backslash A}$ well-defined and it is readily seen that $h$ is the desired f.p. homeomorphism of $E \backslash A$ onto $E \backslash A^{\prime}$. Details may be found in [5] or [8] with the only changes required being the use of f.p. information contained in the fibered $Q$-manifold preliminaries of $\$ 2$.

The following is an immediate

COROLlARY. Let $A$ be a compact sliced Z-set of the compact $Q$-manifold bundle $p: E \rightarrow B$. Suppose for every $b \in B$ there exists an open set $U$ containing $b$ and a compact sliced $Z$-set, $A^{\prime}$, of $E$ which has the same relative 
sliced shape as $A$ and whose complement in $E$ over $U$ is a product, i.e. $p^{-1}(U) \backslash A^{\prime}$ is $f$.p. homeomorphic to $U \times F$ for some $F$. Then $\left.p\right|_{E \backslash A}$ : $E \backslash A \rightarrow B$ is a bundle.

Another direct application of the Sliced Shape Complement Theorems is the

Proof of the boundary classification theorem. First suppose that $A^{\prime}$ is a boundary for $E \stackrel{p}{\rightarrow} B$. Write $N=E \cup A$ and $N^{\prime}=E \cup A^{\prime}$ for our two bundle compactifications. Replace $N$ by $N \times[0,1]$ where $N \times$ $[0,1] \stackrel{\text { proj }_{N}}{\rightarrow} N \stackrel{p}{\rightarrow} B$ is our new bundle map and we use fibered $Z$-set unknotting to have $A \subset N \times\{0\}$. Since $N \times[0,1]-A \cong \cong_{\text {f.p. }} E \cong \cong_{\text {f.p. }} N^{\prime}-A^{\prime}$ there exists a f.p. homeomorphism $h: N \times[0,1]-A \rightarrow N-A^{\prime}$. Also fibered mapping replacement allows us to regard $N \times\{1\}$ as a sliced $Z$-set in $B \times Q$. Define $E_{1}$ to be $(B \times Q) \cup(N \times[0,1])$ with the sewing along $N \times\{1\}$ and $E_{2}$ to be $(B \times Q) \cup N^{\prime}$ sewn along $h(N \times\{1\})$. Then $E_{1} \cong{ }_{\text {f.p. }} E_{2}$ since using fibered collaring they are both easily seen to be f.p. homeomorphic to $B \times Q$. Finally, noting that $A$ and $A^{\prime}$ are sliced $Z$-sets in $E_{1}$ and $E_{2}$ with $E_{1}-A \cong$ f.p. $E_{2}-A^{\prime}$, the sliced shape complement theorem gives us the desired sliced shape equivalence of $A$ and $A^{\prime}$.

For the other direction we let $A$ be a sliced boundary of $E$ and $A^{\prime}$ be of the same sliced shape as $A$. As above, we have a compactification of $E$ given by $E \cup A \cong \cong_{\text {f.p. }} N \times[0,1]$ with $A \subset N \times\{0\}$ and we again consider $E_{1}=(B \times Q) \cup(N \times[0,1])$. When we take a sliced $Z$-embedding of $A^{\prime}$ in $E_{1}$ the sliced shape complement theorem gives us a f.p. homeomorphism $h$ of $E_{1}-A$ onto $E_{1}-A^{\prime}$ (recall that $E_{1} \cong$ f.p. $B \times Q$ ). Then $h(N \times[0,1]-A) \cup A^{\prime}$ gives us a bundle compactification of $E$ with $A^{\prime}$ as the boundary.

As noted previously, Mardešić and Segal offer an alternative approach to shape theory in the unsliced case, see [25]. We briefly discuss the extension of their approach to the sliced category because it makes quick work of demonstrating that two spaces have the same shape later in this paper. Since we are here concerned only with metric spaces we shall not require the generality of arbitrary directed sets with which to index our ANR-systems. Rather we content ourselves with ANR-sequences.

An ANR-sequence $\left(X, X_{0}\right)=\left\{\left(X, X_{0}\right)_{n}, p_{n n+1}\right\}$ consists of an inverse system of pairs of compact absolute neighborhood retracts (ANR's) for metric spaces with bonding maps $p_{n n+1}:\left(X, X_{0}\right)_{n+1} \rightarrow\left(X, X_{0}\right)_{n}$ which are continuous maps of pairs. To incorporate the notion of slicedness over 
a base $B$ we additionally require, for each $n=1,2, \ldots$, a projection map $p_{n}: X_{n} \rightarrow B$ and that bonding maps commute with these projections, i.e. $p_{n} \circ p_{n n+1}=p_{n+1}$ for all $n$. Given a compact pair $\left(X, X_{0}\right)$ we can find an associated ANR-sequence $\left(\underline{X, X_{0}}\right)$ where $\left(X, X_{0}\right)=\underset{\lim }{\leftarrow}\left(X, X_{0}\right)_{n}$. This associated ANR-sequence is unique up to homotopy type as we shall explain and, hence, determines a well-defined property of the space, its relative sliced shape.

$A$ map between ANR-sequences sliced over $B$, denoted $f:\left(X, X_{0}\right) \rightarrow$ $\left(Y, Y_{0}\right)=\left\{\left(Y, Y_{0}\right)_{n}, q_{n n+1}\right\}$, consists of an increasing function $\overline{f \text { from the }}$ natural numbers to itself and a collection of f.p. maps $f_{n}:\left(X, X_{0}\right)_{f(n)} \rightarrow$ $\left(Y, Y_{0}\right)_{n}$ such that there exists a f.p. homotopy of pairs

$$
H: q_{n-1 n} f_{n} \simeq f_{n-1} p_{f(n-1) f(n)}
$$

where

$$
f(n-1) f(n)=p_{f(n-1), f(n-1)+1} \circ p_{f(n-1)+1, f(n-1)+2} \circ \cdots \circ p_{f(n)-1, f(n)} \text {. }
$$

The identity map $\underline{l}\left(X, X_{0}\right):\left(X, X_{0}\right) \rightarrow\left(X, X_{0}\right)$ is given by $1(n)=n$ and $1_{n}=\mathrm{id}_{\left(X, X_{0}\right)_{n}}$. Two maps of ANR-sequences, $\underline{f}, \underline{g}:\left(X, X_{0}\right) \rightarrow\left(Y, Y_{0}\right)$, are homotopic, denoted $\underline{f} \simeq \underline{g}$, if for every integer $n$ there exists an integer $m$ such that $m \geq f(n), g(n)$ and $f_{n} p_{f(n) m} \simeq_{\text {f.p. }} g_{n} p_{g(n) m}$. Finally, $f:\left(X, X_{0}\right)$ $\rightarrow\left(Y, Y_{0}\right)$ is a homotopy equivalence if there exists a map of ANR-sequences $\underline{g}:\left(Y, Y_{0}\right) \rightarrow\left(X, X_{0}\right)$ with $\underline{g f} \simeq \underline{l}_{X}$ and $\underline{f g} \simeq \underline{l}_{Y}$.

The equivalence of the two approaches is asserted in the following

TheOREM. Let $A, A^{\prime} \subset E$ be compacta and $p: E \rightarrow B$ a $Q$-manifold bundle. $A$ and $A^{\prime}$ have the same relative sliced shape in the sense of Borsuk if and only if they have the same relative sliced shape in the sense of ANR-sequences.

The proof of Mardešić and Segal works in the sliced case if we merely carry along the necessary fibered information. Note that the main ingredient of their proof is the homotopy extension theorem and so we provide the following fibered version.

Fibered Homotopy Extension Theorem. Let $\left(A, A_{0}\right)$ be a compact ANR pair and $X \stackrel{p}{\rightarrow} B$ a bundle. Suppose $H: A_{0} \times I \rightarrow X$ is a f.p. homotopy such that $H_{0}$ extends to $\tilde{H}_{0}: A \rightarrow X$. Then there exists a f.p. homotopy $\tilde{H}$ : $A \times I \rightarrow X$ extending $\tilde{H}_{0}$ with $\tilde{H}=H$ on $A_{0} \times I$.

The proof is straightforward. Since $A \times I$ is an ANR containing the closed set $T=\left(A_{0} \times I\right) \cup(A \times\{0\})$ there exists an extension of $H \cup \tilde{H}_{0}$ : $T \rightarrow X$ to a neighborhood of $T$. Though not necessarily f.p. this extension 
is almost f.p. by choosing a small neighborhood of $T$ and hence, using the homotopy lifting property, we can make it f.p. From this point the proof is the same as in the unfibered case as $H$ is defined by composing a push of $A \times I$ into the chosen neighborhood with the extension obtained above.

4. Weak proper homotopy equivalences. In the course of establishing the Homotopy Boundary Theorem we take a route in which (a) our homotopies are not proper and (b) our homotopies and even the maps between our original bundle and our target bundle which admits a boundary are not fiber preserving. That loss of control is what makes this and the next section necessary.

A concept of fundamental importance in the study of non-compact spaces is the definition of a proper map. A map $f: X \rightarrow Y$ is proper if for any compactum $C \subset Y, f^{-1}(C)$ is compact. Proper maps $f$ and $g$ are proper homotopic, written $f \simeq_{p} g$, if they are homotopic through a homotopy which is proper. Spaces $X$ and $Y$ are proper homotopy equivalent (p.h.e.) if there exist proper maps $f: X \rightarrow Y$ and $g: Y \rightarrow X$ and proper homotopies of $f \circ g$ and $g \circ f$ to the appropriate identity maps. The constructions which take place in $\$ 6$ yield homotopies which are not proper but merely weakly proper.

A pair of proper maps $f_{1}, f_{2}: X \rightarrow Y$ are weakly proper homotopic if for any compact set $C \subset Y$ there exists a compact set $D \subset X$ and a homotopy $H: f_{1} \simeq f_{2}$ with $H_{t}(X \backslash D) \subset Y \backslash C$ for all $t$. A proper map $f$ : $X \rightarrow Y$ is a weak proper homotopy equivalence if there exists a proper map $g: Y \rightarrow X$ such that $f \circ g$ and $g \circ f$ are weakly proper homotopic to $\mathrm{id}_{Y}$ and $\mathrm{id}_{X}$, respectively. The knowledgeable reader will recognize the close relationship between weak proper homotopy equivalences and the shape theoretic fundamental sequences employed in the preceding section.

The result which allows us to extract proper information from weak proper homotopy equivalences is Edwards and Hastings', [18].

THEOREM. Let $f: X \rightarrow Y$ be a proper map of $\sigma$-compact, locally compact Hausdorff spaces. If $f$ is a weak proper homotopy equivalence then $f$ is weakly proper homotopic to a genuine proper homotopy equivalence.

We remark that if $X$ and $Y$ are finite dimensional polyhedra then $f$ is itself a proper homotopy equivalence by Siebenmann [27]. The additional parameterized information in the following result is what we require. 
ADDENDUM. Along with the hypotheses above suppose that $p: X \rightarrow B$ and $q: Y \rightarrow B$ are any maps.

(1) If $f, g$ and the homotopies to the identity maps can all be chosen to be fiber preserving then $f$ is weakly proper homotopic through f.p. homotopies to a proper fiber homotopy equivalence.

(2) Suppose that for any open cover $\alpha$ of $B$ there exists maps $f^{\prime}$ : $X \rightarrow Y$ and $g^{\prime}: X \rightarrow Y$ such that $f^{\prime}$ and $g^{\prime}$ are weak proper homotopy inverses with (a) $q f \alpha$-close to $p\left(q f \sim^{\alpha} p\right)$ (b) $p g \sim^{\alpha} q$ and (c) the homotopies of the compositions $f^{\prime} g^{\prime}$ and $g^{\prime} f^{\prime}$ to the respective identity are limited by $\alpha$ when mapped to $B$ (by $q$ and $p$ ). Then for any open cover $\beta$ of $B$ we can find $\tilde{f}: X \rightarrow Y$ and $\tilde{g}: Y \rightarrow X$ such that (a) $q \tilde{f} \sim^{\beta} p$ (b) $p g \sim^{\beta} q$ (c) $\tilde{f}$ and $\tilde{g}$ are proper homotopy inverses with the homotopies involved being limited by $\beta$ when measured in $B$.

We shall call $X$ and $Y$ satisfying the hypotheses of (2) weakly proper homotopy equivalent nearly fiberwise and, similarly, $X$ and $Y$ satisfying the conclusion proper homotopy equivalent nearly fiberwise. Addendum (1) follows immediately from the proof of Edwards and Hastings by choosing f.p. maps and noting that the proper homotopy equivalence that they find is fiber preserving. For addendum (2) simply choose homotopies with better and better control towards the end.

5. Proper lifting. In this section we develop methods for turning proper homotopy equivalences between bundles which are nearly fiberwise into proper fiber homotopy equivalences. Dold does this in [17] without the stipulation that maps be proper. The main ingredient in his proof is the homotopy lifting property for bundles. Thus the result which we require and constitutes the main theorem of this section is what we shall refer to as the

Proper Homotopy Lifting TheOrem. Given any bundle $p: E \rightarrow B$ there exists an open cover $\alpha$ of $B$ such that the following holds. Suppose that $H: X \times I \rightarrow B$ and $\tilde{H}_{0}: X \times\{0\} \rightarrow E$ are maps with $\tilde{H}_{0}$ proper and $p \tilde{H}_{0}=H_{0}$. If $H$ is an $\alpha$-homotopy then there exists a proper map $\tilde{H}$ : $X \times I \rightarrow E$ which extends $\tilde{H}_{0}$ and lifts $H$. In other words we can complete the following commutative diagram with the dotted line being a proper map.

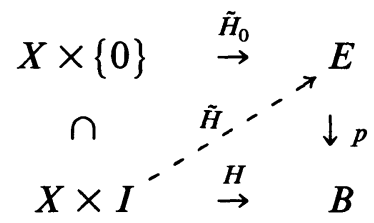


Proof. Let $\beta$ be a cover of $B$ by open sets over which $p$ is trivial. Choose a countable refinement $\left\{U_{i}\right\}_{i=1}^{\infty}$ of $\beta$ which also covers $B$ and with each $\bar{U}_{i}$ compact and each $\bar{U}_{i}$ contained in some open set of $B$ over which $p$ is trivial. Set $\alpha=\left\{U_{i}\right\}_{i=1}^{\infty}$.

We now define a tower of compacta, $\left\{C_{i}\right\}$, whose union is $E$. Begin with $\left\{\hat{C}_{i}\right\}$ where each $\hat{C}_{i}$ is compact, $\hat{C}_{i} \subset{ }_{\text {int }} \hat{C}_{i+1}$ and $\cup_{i=1}^{\infty} \hat{C}_{i}=F$, the fiber of $p: E \rightarrow B$. Define $C_{1}=h_{1}\left(\hat{C}_{1} \times \bar{U}_{1}\right)$ where $h_{i}: F \times \bar{U}_{i} \rightarrow p^{-1}\left(\bar{U}_{i}\right)$ is a homeomorphism arising from the bundle structure of $E$. Assume that $C_{j}$ has been defined for all $j \leq k, k$ some positive integer. From the construction to follow note that the $\hat{C}_{j}$ are renumbered as needed so that we also assume that the sets we refer to below have already been reindexed. Clearly $h_{j}\left(\hat{C}_{k} \times \bar{U}_{j}\right) \cap h_{i}\left(\hat{C}_{k} \times \bar{U}_{i}\right)=C^{i, j}$ is compact, $i$ and $j \leq k$. Set $\hat{C}^{i, j}=p_{F}\left(h_{j}^{-1}\left(C^{i, j}\right)\right) \cup p_{F}\left(h_{i}^{-1}\left(C^{i, j}\right)\right)$ a compactum of $F$. There exists $N>k$ such that $\bigcup_{i, j \leq k} \hat{C}^{i, j} \subset$ int $\hat{C}_{N}$. Reindex the $\hat{C}_{j}$ for $j=k+1$, $k+2, \ldots$ with the new $\hat{C}_{k+i}$ being the old $\hat{C}_{N+i-1}, i=1,2,3, \ldots$ Then we take $C_{k+1}=\bigcup_{i=1}^{k+1} h_{i}\left(\hat{C}_{i} \times \bar{U}_{i}\right)$.

Considering $\tilde{H}_{0}$ as a map from $X$ to $E$ let $W_{1}=\tilde{H}_{0}^{-1}\left(C_{1}\right) \times I$, a compact subset of $X \times I$ since $\tilde{H}_{0}$ is proper. Inductively define a cover of $X \times I$ by compacta $W_{i}$ with $W_{i} \subset$ int $W_{i+1}$ by noting that $\bigcup_{i=1}^{\infty} \tilde{H}_{0}^{-1}\left(C_{i}\right)=$ $X$ and setting $W_{i}=\tilde{H}_{0}^{-1}\left(C_{k}\right) \times I$ for $k$ sufficiently large. Finally, renumber the $C_{i}\left(\right.$ and $\left.\hat{C}_{i}\right)$ so that $W_{i}=\tilde{H}_{0}^{-1}\left(C_{i}\right) \times I$.

Assuming that $H$ is an $\alpha$-homotopy we have, for every $x \in X$, $H(\{x\} \times I) \subset U_{i}$ for some $i$. Then $\{x\} \times I \subset H^{-1}\left(U_{i}\right)$, an open set, and so by the tube lemma there exists an open set $O_{x}^{\prime}$ of $X$ containing $x$ with $O_{x}^{\prime} \times I \subset H^{-1}\left(U_{i}\right)$. Without loss of generality (by intersecting covers) we may assume that the cover $\left\{O_{x}^{\prime} \times I\right\}_{x \in X}$ refines the open cover $\left\{\stackrel{\circ}{W}_{2}, \stackrel{\circ}{W}_{3}\right.$ $\left.-W_{1}, \ldots, \mathscr{W}_{k}-W_{k-2}, \ldots\right\}$ of $X \times I$. Cutting back slightly we can find open sets $O_{x}$, such that for any $x \in X, x \in O_{x} \subset \bar{O}_{x} \subset O_{x}^{\prime}$ with $\bar{O}_{x}$ compact. For every $k=2,3,4, \ldots$ choose a finite refinement of $\left\{O_{x}\right\}_{x \in X}$ which covers the compact set $W_{k}-\stackrel{\circ}{W}_{k-1}$ say $\left\{O_{k,}\right\}_{j=1}^{n_{k}}$ where $O_{k}^{\prime} \times I \subset$ $H^{-1}\left(U_{k_{j}}\right)$ and $O_{k,}^{\prime} \subset \stackrel{\circ}{W+1}_{k+1}-W_{k-3}$ taking $W_{i}=\varnothing$ for $i=0$ or -1 and $k_{j}<k_{j+1}$.

At long last we are in a position to define $\tilde{H}$. Choose Urysohn functions $u_{j}: X \rightarrow[0,1]$ such that $u_{j}\left(\bar{O}_{1_{j}}\right)=1$ and $u_{j}\left(X-O_{1_{j}}^{\prime}\right)=0$ for $1 \leq j \leq n_{1}$. Let $\tau_{k}(x)=\max _{1 \leq j \leq k}\left\{u_{j}(x)\right\}$ and $\tau_{0}(x)=0$ and $X_{k}^{1}=$ $\left\{(x, t) \mid 0 \leq t \leq \tau_{k}(x)\right\} \subset \stackrel{\circ}{W}_{2}$. Set

$$
\tilde{H}(x, t)=h_{1_{k}}\left(p_{F} h_{1_{k}}^{-1} \tilde{H}\left(x, \tau_{k-1}(x)\right), H(x, t)\right)
$$

for $x \in X_{k}^{1}-X_{k-1}^{1}$. This defines $\tilde{H}$ on $X_{n_{1}}^{1}$, which contains $W_{1}$. 
Inductively suppose that $\tilde{H}$ is defined on $X_{n_{k}}^{k}$ containing $W_{k}$. We show how to extend to $X_{n_{k+1}}^{k+1}$ containing $W_{k+1}$. Take Urysohn functions $u_{j}$ : $X \rightarrow\left[\tau_{n_{k}}(x), 1\right]$ such that $u_{j}\left(\bar{O}_{(k+1)_{j}}\right)=1$ and $u_{j}\left(X-O_{(k+1)_{j}}^{\prime}\right)=\tau_{n_{k}}(x)$ where $1 \leq j \leq n_{k+1}$. Set $\tau_{j}(x)=\max _{1 \leq i \leq j}\left\{u_{i}(x)\right\}$ with $\tau_{0}(x)=\tau_{n_{k}}(x)$ and let

$$
X_{1}^{k+1}=\left\{(x, t) \mid \tau_{n_{k}}(x) \leq t \leq \tau_{1}(x)\right\} \subset \stackrel{\circ}{W}_{k+2}-W_{k}
$$

Define

$$
\tilde{H}(x, t)=h_{(k+1)_{i}}\left(p_{F} h_{(k+1)_{i}}^{-1} \tilde{H}\left(x, \tau_{i-1}(x)\right), H(x, t)\right)
$$

for $x \in X_{i}^{k+1}-X_{i-1}^{k+1}\left(X_{0}^{k+1}=X_{n_{k}}^{k}\right)$.

The continuity of $\tilde{H}$ is clear from the formula and the observation that the definition extends $\tilde{H}$ from $X_{i-1}^{k+1}$ to $X_{i}^{k+1}$ (as well as from $X_{n_{k}}^{k}$ to $X_{1}^{k+1}$ ) continuously. The last coordinate in the formula assures us that $\tilde{H}$ is a lift of $H$. For the fact that $\tilde{H}$ is proper, note that $\tilde{H}$ is close to the proper map $\tilde{H}_{0} \circ \Pi_{X}: X \times I \rightarrow E$. Specifically, the two maps are close as measured by an open cover of rings formed from the $C_{i}$, i.e. open sets of the form int $C_{k+1}-C_{k}$.

The (non-proper) homotopy lifting property of a map $p: E \rightarrow B$ states that given a homotopy $H: X \times I \rightarrow B$ such that one end lifts, i.e. there is a map $\tilde{H}_{0}: X \times\{0\} \rightarrow E$ with $p \tilde{H}_{0}=H_{0}$, there exists a lift of the homotopy extending $\tilde{H}_{0}$, a map $\tilde{H}: X \times I \rightarrow E$ with $p \tilde{H}=H$ and $\tilde{H} \mid X \times$ $\{0\}=\tilde{H}_{0}$. Dold, Theorem 6.1 in [17], makes repeated use of such lifts to prove the following

TheOReM. Let $p: E \rightarrow B$ and $p^{\prime}: E^{\prime} \rightarrow B$ be spaces over $B$ which have the homotopy lifting property, e.g. bundles. Then if a f.p. map $f: E \rightarrow E^{\prime}$ is an ordinary homotopy equivalence it is a fiber homotopy equivalence.

Making use of our Proper Homotopy Lifting Theorem in Dold's proof easily yields the following result.

TheOREM. Let $p: E \rightarrow B$ and $p^{\prime}: E^{\prime} \rightarrow B$ be bundles. There exists an open cover $\alpha$ of $B$ such that if $f: E \rightarrow E^{\prime}$ is a proper $f . p$. map which is $a$ proper homotopy equivalence through homotopies limited by $\alpha$ when measured in $B$ then $f$ is a proper fiber homotopy equivalence.

Proof. Choose the open cover $\alpha$ from the Proper Lifting Theorem. Let $g^{\prime}$ be a proper homotopy inverse of $f$ and $H: E^{\prime} \times I \rightarrow E^{\prime}$ a proper homotopy with (i) $H_{0}=f g^{\prime}$ (ii) $H_{1}=\mathrm{id}_{E^{\prime}}$ and (iii) for every $e^{\prime} \in E^{\prime}$ there exists $0 \in \alpha$ such that $p^{\prime} H\left(\left\{e^{\prime}\right\} \times I\right) \subset 0$. 
The homotopy $p^{\prime} H: E^{\prime} \times I \rightarrow B$ has a proper lift to $E$ which we call $G: E^{\prime} \times I \rightarrow E$ satisfying $G_{0}=g^{\prime}$. Setting $g=G_{1}$ we proceed to show that $g$, which is fiber preserving, is a proper fiber homotopy inverse of $f$.

Define a homotopy $\tilde{H}: E^{\prime} \times[0,2] \rightarrow E^{\prime}$ from $f g$ to $\mathrm{id}_{E^{\prime}}$ by the formula

$$
\tilde{H}\left(e^{\prime}, s\right)= \begin{cases}f G\left(e^{\prime}, 1-s\right) & \text { for } 0 \leq s \leq 1 \\ H\left(e^{\prime}, s-1\right) & \text { for } 1 \leq s \leq 2\end{cases}
$$

$\tilde{H}$ then is proper and, though not vertical, satisfies

$$
p^{\prime} \tilde{H}\left(e^{\prime}, s\right)=p^{\prime} \tilde{H}\left(e^{\prime}, 2-s\right) .
$$

Exploiting this last equation we lift the homotopy $h: E^{\prime} \times[0,2] \times[0,1]$ $\rightarrow B$ given by

$$
h\left(e^{\prime}, s, t\right)= \begin{cases}p^{\prime} \tilde{H}\left(e^{\prime}, s\right) & \text { for } s \leq 1-t \\ p^{\prime} \tilde{H}\left(e^{\prime}, 1-t\right)=p^{\prime} \tilde{H}\left(e^{\prime}, 1+t\right) & \text { for } 1-t \leq s \leq 1+t \\ p^{\prime} \tilde{H}\left(e^{\prime}, s\right) & \text { for } s \geq 1+t\end{cases}
$$

to a proper homotopy, $\hat{H}: E^{\prime} \times[0,2] \times[0,1] \rightarrow E^{\prime}$ with $\hat{H}\left(e^{\prime}, s, 0\right)=$ $\tilde{H}\left(e^{\prime}, s\right)$. It is then an easy check to see that going around three sides of $\hat{H}$, i.e. letting $F: E^{\prime} \times[0,4] \rightarrow E^{\prime}$ be defined by

$$
F\left(e^{\prime}, t\right)= \begin{cases}H\left(e^{\prime}, 0, t\right) & \text { for } t \leq 1 \\ \hat{H}\left(e^{\prime}, t-1,1\right) & \text { for } 1 \leq t \leq 3 \\ \hat{H}\left(e^{\prime}, 2,4-t\right) & \text { for } 3 \leq t\end{cases}
$$

gives a proper, f.p. homotopy between $f g$ and $\mathrm{id}_{E^{\prime}}$.

We can now apply the same argument with $g$ in place of $f$ and obtain a proper map $f^{\prime}: E \rightarrow E^{\prime}$ such that we have a proper homotopy $g f^{\prime}$ $\simeq_{\text {f.p. }} \mathrm{id}_{E}$. Consequently the string of proper, f.p. homotopies

$$
g f \simeq(g f)\left(g f^{\prime}\right)=g(f g) f^{\prime} \simeq g f^{\prime} \approx \mathrm{id}_{E}
$$

establishes the result.

6. Homotopy boundary theorem. We are given a non-compact $Q$-manifold bundle $p: M \rightarrow B$ which has sliced finite type and is slicewise tame at $\infty$. Let $q: N \rightarrow B$ be a compact $Q$-manifold bundle guaranteed by the former hypothesis with f.p. homotopy inverses $f: M \rightarrow N$ and $g: N \rightarrow M$. Using the sliced tameness at $\infty$ we can find a neighborhood system of $\infty$, $\left\{M-M_{i}\right\}_{i=1}^{\infty}$, i.e. compact sets $M_{i} \subset \mathrm{M}$ with $M_{i} \subset$ int $M_{i+1}$ and $\bigcup_{i=1}^{\infty} M_{i}$ $=M$, and finite polyhedra $K_{i}, i=1,2,3, \ldots$, and a f.p. homotopy 
commutative diagram

(*)

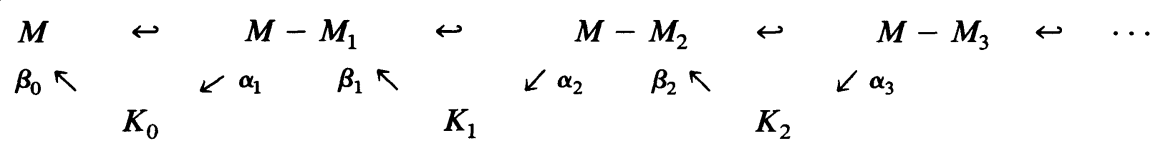

Set $\hat{A}=\lim _{\leftarrow}\left\{K_{i}, \alpha_{i}, \beta_{i}\right\}$ recalling that this means that

$\hat{A}=\left\{\left(k_{0}, k_{1}, k_{2}, \ldots\right) \in \prod_{i=0}^{\infty} K_{i} \mid\left(\alpha_{i+1} \beta_{i+1}\right)\left(k_{i+1}\right)=k_{i}\right.$ for $\left.i=0,1,2, \ldots\right\}$.

Approximate $f \beta_{1} \pi_{K_{1}}: \hat{A} \rightarrow N, \pi_{K_{1}}$ denoting projection to $K_{1}$, by a sliced Z-embedding $\phi: \hat{A} \rightarrow N$. Letting $A=\phi(\hat{A})$ we now have our target, $N-A$, which we will show is proper fiber homotopy equivalent to $M$.

An intermediate space is useful in establishing the desired proper fiber homotopy equivalence. In Chapman-Siebenmann, [14], a $Q$-manifold with finite type which is tame at $\infty$ is shown to be proper homotopy equivalent to a certain mapping telescope, $\operatorname{Map}(\sigma)$ where $\sigma$ is the inverse sequence of compact polyhedra $\left\{K_{i}, \alpha_{i} \beta_{i}\right\}$. Chapman's observation that $\hat{A} \times Q=\underset{\leftarrow}{\lim } \sigma \times Q$ is the natural boundary of $\operatorname{Map}(\sigma) \times Q$ is the heart of [6] and also appears in the more detailed analysis of [14]. We briefly review the constructions of Chapman and Siebenmann for the convenience of the reader and to justify the additional observations which we require for the fibered situation.

The f.p. homotopy ladder, $(*)$, is formed with care in order to have the $M_{i}$ 's clean submanifolds of $M$, i.e. each $M_{i}$ is a closed $Q$-manifold as is its topological frontier $\partial M_{i}$ which is also collared in both $M_{i}$ and the closure of $M-M_{i}$. The finite polyhedra $K_{i}$ are for convenience considered to be contained in $\operatorname{int}\left(M_{i+1}\right)-M_{i}$ with the maps $\beta_{i}: K_{i} \rightarrow M-M_{i}$ being inclusions. Let $\alpha_{i}^{t}$ : $M$ - int $M_{i} \rightarrow M$ - int $M_{i}$ be homotopies with $\alpha_{i}^{0}=\mathrm{id}$ and $\alpha_{i}^{1}=\alpha_{i}$ noting that these are f.p. in our problem. By the sliced finite type of $M$ we also can assume the existence of a f.p. retraction $\alpha_{0}^{t}:$ id $\simeq \alpha_{0}^{1}$ where $\alpha_{0}^{1}$ maps $M$ into a compact polyhedron $K_{0} \subset$ int $M_{1}$. The locally compact $\operatorname{space} \operatorname{Map}(\sigma)$ where $\sigma=\left\{K_{i},\left.\alpha_{i}^{1}\right|_{K_{i}}\right\}_{i=0}^{\infty}$ is the infinite union of the mapping cylinders $M\left(\alpha_{i}^{1}\right)$ sewn together in a natural way along their ends. Recall that $M\left(\alpha_{i}^{1} \mid\right), \alpha_{i}^{1} \mid: K_{i} \rightarrow K_{i-1}$, is the disjoint union $K_{i} \times[0,1] \amalg K_{i-1}$ with $\alpha_{i}^{1}(k)$ identified with $(k, 1)$ for $k \in K_{i}$.

Weak proper homotopy inverses $\bar{\alpha}: \operatorname{Map}(\sigma) \rightarrow M$ and $\bar{\beta}: M \rightarrow$ $\operatorname{Map}(\sigma)$ are then defined on compact pieces as follows. $\bar{\alpha} \mid: M\left(\alpha_{i}^{1} \mid\right) \rightarrow$ $M \backslash$ int $M_{i}$ is just the map which sends $(x, t)$ to $\alpha_{i}^{t}(x)$ for $(x, t) \in$ $K_{i} \times[0,1]$ and $x$ to itself for $x \in K_{i-1} \cdot \bar{\beta} \mid: M_{i+1}-$ int $M_{i} \rightarrow M\left(\alpha_{i}^{1} \mid\right)$ is 
$\alpha_{i}^{1}$ on $M_{i+1}-\left[\partial\left(M_{i+1}\right) \times[0,2) \cup\right.$ int $\left.M_{i}\right]$, where $\partial\left(M_{i+1}\right) \times[0,2] \subset$ $M_{i+1}-\left(\operatorname{int}\left(M_{i}\right) \cup K_{i}\right)$ is a collar of $\partial M_{i+1}$ in $M_{i+1}$ with $\partial\left(M_{i+1}\right) \times\{0\}=$ $\partial M_{i+1}$, and

$$
\bar{\beta}(x, t)= \begin{cases}\alpha_{i}^{1} \alpha_{i+1}^{2-t}(x) & \text { for } x \in \partial\left(M_{i+1}\right) \times[1,2] \\ \left(\alpha_{i+1}^{1}(x), t\right) \in K_{i+1} \times[0,1) & \text { for } x \in \partial\left(M_{i+1}\right) \times[0,1) .\end{cases}
$$

In the fibered setting one should recognize that $\bar{\beta}$ and $\bar{\alpha}$ as well as the homotopies which deform the compositions $\bar{\beta} \circ \bar{\alpha}$ and $\bar{\alpha} \circ \bar{\beta}$ to the identity maps are all nearly fiberwise by choosing short collars. Therefore, plugging this information into our fibered version of Edwards and Hastings yields

Proposition 1. There exists a proper homotopy equivalence, $k: M \rightarrow$ $\operatorname{Map}(\sigma)$, which is nearly fiberwise.

We remark that the loss of absolute fiber control above was due to the fact that our collars were not fibered collars. Another difficulty with using $\operatorname{Map}(\sigma)$, or $\operatorname{Map}(\sigma) \times Q$, as a bridge between $M$ and $N-A$ is that $\operatorname{Map}(\sigma)$ is not a bundle and so we again are forced to settle for near fiber control below.

Proposition 2. $N-A$ is proper homotopy equivalent nearly fiberwise to $\operatorname{Map}(\sigma) \times Q$.

Proof. Setting $X=\operatorname{Map}(\sigma) \cup \hat{A}$ we shall actually prove the equivalence between $N \times I-A \times 0$ and $X \times Q-\hat{A} \times 0$. Replacing $N-A$ by the f.p. homeomorphic $Q$-manifold $N \times I-A \times 0$ is a standard device using fibered stability and $Z$-set unknotting. On the other hand we note that $\hat{A} \times 0$ and $\hat{A} \times Q$ clearly have the same relative shape in $X \times Q$ and so $X \times Q-\hat{A} \times 0$ is homeomorphic to $X \times Q-\hat{A} \times Q=\operatorname{Map}(\sigma) \times Q$. Moreover, examination of the proof of the Complement Theorem which provides the homeomorphism reveals that it is nearly fiberwise.

The inclusion $\hat{A} \stackrel{i}{\hookrightarrow} X$ and the composition

$$
\hat{A} \stackrel{\phi}{\rightarrow} N \stackrel{g}{\rightarrow} M \stackrel{k}{\rightarrow} \operatorname{Map}(\sigma) \stackrel{i}{\rightarrow} X
$$

are homotopic nearly fiberwise. For by closeness $\phi \simeq f \beta_{1} \pi_{K_{1}}$ and $i \simeq i \pi_{K}$, for $j$ sufficiently large. We shall write $f i \pi_{K_{1}}$ for $f \beta_{1} \pi_{K_{1}}$ since $\beta_{1}$ is now the inclusion map and remark that the product topology on $\Pi_{i=1}^{\infty} K_{i}$ provides the closeness in the latter homotopy with $\pi_{K}$ understood to be 
$\hat{A} \subset \Pi_{i=1}^{\infty} K_{i} \stackrel{\text { proj }}{\rightarrow} K_{j} \hookrightarrow \operatorname{Map}(\sigma)$. Thus we have

$$
\begin{aligned}
i k g \phi & \simeq_{\text {f.p. }} i k g f_{i} \pi_{K_{i}} \simeq_{\text {f.p. }} i k i \pi_{K_{1}}=i k i k \alpha_{2} \alpha_{3} \cdots \alpha_{j+1} \alpha_{j+1} \pi_{K_{j+1}} \\
& \simeq_{\text {f.p. }} i k i \pi_{K_{J+1}} \simeq i \pi_{K_{J}} \simeq i \pi_{K_{j}} .
\end{aligned}
$$

Approximate $i_{0} i k g: N \rightarrow X \times Q$ by a $Z$-embedding $\eta$ where $i_{0}: X \rightarrow X \times$ $Q$ is defined by setting $i_{0}(x)=(x, 0)$. Since $\eta \phi \simeq i_{0} i k g \phi \simeq i_{0} i$ we may assume that $\eta \phi=i_{0} i$.

Next let $\mu: N \times I \rightarrow M(\eta)$ be the quotient map and $c: M(\eta) \rightarrow X \times Q$ the collapse to the base. Since $c$ is a CE map (for $x \in X \times Q, c^{-1}(x)$ is either a point or the unit interval) it is close to a homeomorphism $\hat{c}$ : $M(\eta) \rightarrow X \times Q$ which unknotting again we can adjust so that $A \times 0 \subset N$ $\times 0$ is mapped onto $\hat{A} \times 0 \subset X \times 0$. Then $\hat{c} \mu \mid: N \times I-A \times 0 \rightarrow X \times Q$ $-\hat{A} \times 0$ is a homeomorphism near $\infty$ and an ordinary homotopy equivalence and thus a proper homotopy equivalence. Though $\operatorname{Map}(\sigma)$ not being a bundle prevents the use of fibered results the maps are close to the original nearly f.p. composition $i_{0} i k g$ and so also using $Z$-set unknotting with control we get that the equivalence is nearly fiberwise.

Consequently it is clear that $M$ and $N-A$ are proper homotopy equivalent nearly fiberwise. To achieve fiber control we establish the following

Proposition 3. $\left.q\right|_{N-A}: N-A \rightarrow B$ is a bundle.

Proof. According to the corollary to the sliced shape complement theorem we need but to show that, for each small open set $U$ of $B, A$ has the same sliced relative shape as some compact sliced $Z$-set whose complement in $N$ is a product over $U$. This is accomplished in two steps. First we prove that any $A^{\prime}$ defined similarly to $A$ but with possibly different choices of $\sigma$ and appropriate sliced $Z$-embedding of $\lim \sigma$ has the same sliced relative shape (in $N$ ) as $A$. Then for any $b \in B$ we find an open set $U$ containing $b$ and construct a homotopy ladder which yields a sliced Z-set $A^{\prime}$ with $p^{-1}(U) \cap\left(N-A^{\prime}\right) \approx_{\text {f.p. }} U \times F$ for some $F$.

Step 1. Assume that two different choices of inverse sequences of compact polyhedra obtained by the sliced tameness of $\infty$ hypothesis on $p$ : $M \rightarrow B$ have been made. That is, we have f.p. homotopy commutative diagrams

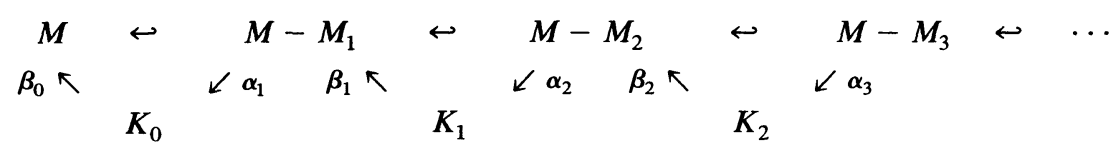


and

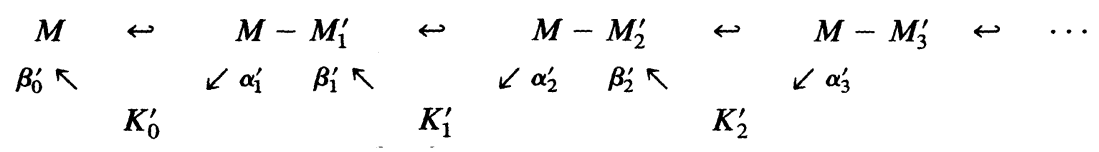

which give rise to compacta $\hat{A}=\lim _{\leftarrow}\left\{K_{i}, \alpha_{i} \beta_{i}\right\}$ and $\hat{A}^{\prime}=\lim _{\leftarrow}\left\{K_{i}^{\prime}, \alpha_{i}^{\prime} \beta_{i}^{\prime}\right\}$. By approximating the maps $\beta_{i}: K_{i} \rightarrow M-M_{i}$ and $f: M \rightarrow N$ by sliced $Z$-embeddings we assume that $K_{i} \subset M-M_{i} \subset N\left(M_{0}=\phi\right)$. Let $\hat{\alpha}_{i}$ : $\left(N, K_{i}\right) \rightarrow\left(N, K_{i-1}\right)$ be a f.p. extension of $\alpha_{i}: M-M_{i} \rightarrow N$ with $\hat{\alpha}_{i}$ $\simeq_{\text {f.p. }}$ id. Consider the f.p. inverse sequence of pairs

$$
\left(N, K_{0}\right) \stackrel{\hat{\alpha}_{1}}{\leftarrow}\left(N, K_{1}\right) \stackrel{\hat{\alpha}_{2}}{\leftarrow}\left(N, K_{2}\right) \leftarrow \cdots .
$$

It is a simple matter to find increasing sequences $\left\{n_{i}\right\}$ and $\left\{n_{i}^{\prime}\right\}$ and shuffle sequences to get the following diagram which commutes up to f.p. homotopy

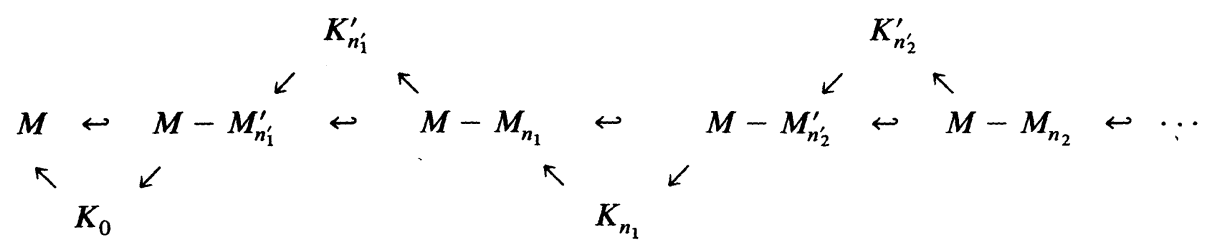

This demonstrates that $A$ and $A^{\prime}$ have the same sliced relative shape in $N$ by the ANR-system approach and completes Step 1.

Step 2. Let $b \in B$ be given. Choose an open set $U \subset B$ such that

(i) $b \in U$,

(ii) $\partial \bar{U}$ is collared in $B-U$, we write simply that $\partial \bar{U} \times[0,2)$ is an open set of $B-U$ with $\partial \bar{U} \equiv \partial \bar{U} \times\{0\}$,

(iii) $p$ is trivial over $U \cup(\partial \bar{U} \times[0,1])=\tilde{U}$ and

(iv) $\tilde{U}$ contracts to $b$ say by the homotopy $H: \tilde{U} \times I \rightarrow \tilde{U}$ with $H_{0}=$ id and $H_{1}(\tilde{U})=\{b\}$.

Form a new ladder

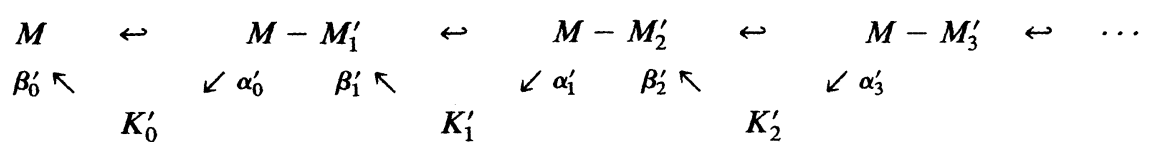

from our original ladder (*) as follows. First extend $H$ to $\hat{H}: B \times I \rightarrow B$ by feathering $H$ to the identity outside of $\tilde{U}$. For a formula we may use

$$
\hat{H}_{t}(u)= \begin{cases}H_{t}(u) & \text { for } u \in U \\ H_{t(1-s)}(u) & \text { for } u=\left(u^{\prime}, s\right) \in \partial \bar{U} \times[0,1] \\ u & \text { for } u \in B-\tilde{U} .\end{cases}
$$


Now, letting $h: \tilde{U} \times F \rightarrow p^{-1}(\tilde{U})$ be a f.p. homeomorphism define $M-M_{i}^{\prime}$ to be $M-M_{i}$ outside of $p^{-1}(\tilde{U})$ and a product over $U$. Specifically, set

$$
\begin{aligned}
M-M_{i}^{\prime}= & \left(p^{-1}(B-\tilde{U}) \cap\left(M-M_{i}\right)\right) \\
& \cup\left\{h\left(\pi_{F} h^{-1}\left[p^{-1}\left(\hat{H}_{1}(U)\right) \cap\left(M-M_{i}\right)\right], u\right) \mid u \in \tilde{U}\right\} .
\end{aligned}
$$

In a similar way one can define the $K_{i}^{\prime}$ to be $K_{i} \cap \beta_{i}^{-1} p^{-1}(B-\tilde{U})$ outside of $\beta_{i}^{-1} p^{-1}(\tilde{U})$ and products over $U$ and the $\alpha_{i}^{\prime}$ and $\beta_{i}^{\prime}$ are defined naturally from the $\alpha_{i}$ and $\beta_{i}$. The net effect is that we have stretched what the original diagram saw over $p^{-1}(b)$ to a product over $p^{-1}(U)$.

Clearly, $\hat{A}^{\prime}=\lim _{\leftarrow}\left\{K_{i}^{\prime}, \alpha_{i}^{\prime} \beta_{i}^{\prime}\right\}$ is a product over $p^{-1}(U)$. Choosing an appropriate copy $\overleftarrow{A^{\prime}}$ of $\hat{A}^{\prime}$ in $N$ we see that over $q^{-1}(U)$ we shall be removing a product when we form $N-A^{\prime}$. We take a moment to show why $q^{-1}(U) \cap\left(N-A^{\prime}\right)$ is a product. Consider the f.p. map $\left.f\right|_{p^{-1}(\tilde{U})}$ : $F \times \tilde{U} \rightarrow F^{\prime} \times \tilde{U}$ where we choose $\tilde{U}$ shall enough so that $q$ is trivial over it and suppress the homeomorphisms resulting from the local triviality of $p$ and $q$. Define a f.p. homotopy $f_{t}: F \times U \rightarrow F^{\prime} \times \tilde{U}$ by setting $f_{t}(x, u)=$ $\left(p_{F^{\prime}} f\left(x, \hat{H}_{t}(u)\right), u\right)$. Note that

(i) $f_{0}=f$,

(ii) $f_{t}$ extends to all of $M$ by letting $f_{t}=f$ on $M-(F \times \tilde{U})$, and

(iii) $f_{1}(x, u)=\left(p_{F^{\prime}} f(x, b), u\right)$ for all $(x, u) \in F \times U$, i.e. $f_{1}$ is a product map on $F \times U$. Approximate the maps $f \beta_{1}^{\prime} \pi_{K_{1}^{\prime}}$ and $f_{1} \beta_{1}^{\prime} \pi_{K_{1}^{\prime}}$ by sliced Z-embeddings to get copies of $\hat{A}^{\prime}, A^{\prime}$ and $A^{\prime \prime}$, respectively, in $N$. $N-A^{\prime \prime}$ is a product over $U$ since $\hat{A}^{\prime}$ is and $f_{1}, \beta_{1}^{\prime}$ and $\pi_{K_{1}^{\prime}}$ are all product maps there. Then by fibered $Z$-set unknotting $N-A^{\prime}$ and $N-A^{\prime \prime}$ are fiber homeomorphic.

Proof of the homotopy boundary theorem. We have established that $M$ and $N-A$ are bundles which are proper homotopy equivalent nearly fiberwise. By our proper version of Dold's theorem we need only find a f.p. proper map $\tilde{f}: M \rightarrow N-A$ which is a homotopy equivalence through homotopies which are small when measured in $B$. But this is easy. For let $f: M \rightarrow N-A$ be a proper homotopy equivalence that is nearly fiberwise. Since $B$ is a polyhedron and $p$ is close to $q f$ the two maps are homotopic via a small homotopy. Choose a homotopy inverse of $f, g: N-A \rightarrow M$, and use the Proper Homotopy Lifting Theorem to left $H: q f g f \simeq p$ to $\tilde{H}$ : $M \times I \rightarrow N-A$ with $\tilde{H}_{0}=f g f$. Then $\tilde{H}_{1}: M \rightarrow N-A$ is f.p. and proper and fulfills our needs since $\tilde{H}_{1} \simeq f g f \simeq f$, the homotopies being f.p. and nearly f.p., respectively. 
7. Theorem 2. We are given that $f: N \rightarrow N^{\prime}$ is a proper fiber homotopy equivalence between bundles $q: N \rightarrow B$ and $q^{\prime}: N^{\prime} \rightarrow B$ and that $N$ and $N^{\prime}$ admit boundaries. Let $A$ and $A^{\prime}$ be sliced $Z$-sets (in $N \cup A$ and $N^{\prime} \cup A^{\prime}$ ) which compactify $N$ and $N^{\prime}$ where we retain $q$ and $q^{\prime}$ as bundle maps. Let $F_{t}$ be an instantaneous f.p. homotopy push of $N \cup A$ off of $A$ as in Lemma 1 of $\S 3$. Approximate

$$
N \cup A \stackrel{F_{1}}{\rightarrow} N \stackrel{f}{\rightarrow} N^{\prime} \hookrightarrow N^{\prime} \cup A^{\prime}
$$

by a sliced Z-embedding $f^{\prime}: N \cup A \rightarrow N^{\prime} \cup A^{\prime}$.

Claim. $f^{\prime}(A)$ and $A^{\prime}$ are slicewise relatively fundamentally equivalent in $N^{\prime} \cup A^{\prime}$.

The method of proof is much like that used in $\$ 3$ and so we just indicate the construction of the slicewised relative fundamental sequences between $f^{\prime}(A)$ and $A^{\prime}$. Let $f^{\prime}(N \cup A) \times[0,1)$ be a fibered collar of $f^{\prime}(N \cup A)$ in $N^{\prime} \cup A^{\prime}$ with $f^{\prime}(N \cup A)=f^{\prime}(N \cup A) \times\{0\}$. Define $\underline{f}=\left\{f_{k}\right.$ : $\left.f^{\prime}(N \cup A) \times[0,1) \rightarrow N^{\prime}\right\}$ by $f_{k}=f F_{1 / k}\left(f^{\prime}\right)^{-1} \pi_{f^{\prime}(N \cup A)}$, where $\pi$ denotes projection, and $\underline{g}=\left\{g_{k}: N^{\prime} \cup A^{\prime} \rightarrow N^{\prime}\right\}$ by $g_{k}=f^{\prime} g G_{1 / k}$ where $g$ is a proper fiber homotopy inverse of $f$ and $G_{t}$ is a homotopy push off of $A^{\prime}$.

Therefore, by the sliced shape complement theorem, there is a f.p. homeomorphism $h:\left(N^{\prime} \cup A^{\prime}\right)-f^{\prime}(A) \rightarrow N^{\prime}$ with $h \simeq_{\text {f.p. }}$ id. Then $h \mid: f^{\prime}(N$ $\cup A) \times[0,1 / 2]-f^{\prime}(A) \rightarrow N^{\prime}$ is a f.p. homeomorphism near $\infty$ and by fibered stability and $Z$-set unknotting there exists a f.p. homeomorphism $s: \quad N \rightarrow(N \cup A) \times[0,1 / 2]-A \times\{0\}$. Then $\phi=h\left(f^{\prime} \times\right.$ id $) s$ is a f.p. homeomorphism near $\infty$ between $N$ and $N^{\prime}$. It remains to be shown that $\phi$ is proper fiber homotopic to $f$. That $\phi$ and $f$ are homotopic through a f.p. homotopy is easy, indeed

$$
\phi=h\left(f^{\prime} \times \mathrm{id}\right) s \simeq_{\text {f.p,p }} h f^{\prime} \simeq_{\text {f.p. }} f^{\prime} \simeq_{\text {f.p. }} f F_{1} \simeq_{\text {f.p. }} f .
$$

A slightly more delicate analysis is needed to achieve the proper requirement; the homotopy above is not proper. Part of the problem is the fact that our homotopy push of $N \cup A$ off of $A$, namely $F_{t}$, is decidedly not proper when restricted to $N$.

Knowledge of the construction of the homeomorphism $h$ from the sliced shape complement theorem is what enables us to find a proper homotopy. Rather than getting stuck in the morass of the proof of $\$ 3$ we indicate how to view $h$ more simply, basically by combining the constructions of the complement theorem with those of the lemma preceding it as Chapman does in the unfibered case in [8]. Briefly, there exists an increasing sequence of positive integers $\left\{N_{i}\right\}_{i=1}^{\infty}$ and sliced Z-embeddings 
$\alpha_{2 n+1}$ and $\alpha_{2 n}$ approximating $f_{N_{2 n+1}}$ and $g_{N_{2 n}}$, respectively. Then by fibered $Z$-set unknotting and extending by the identity outside of smaller and smaller neighborhoods of $f^{\prime}(A)$ and $A^{\prime}$, we get f.p. homeomorphisms $h_{2 n+1}, \quad h_{2 n}^{-1}: \quad N^{\prime} \rightarrow N^{\prime}$ extending $\alpha_{2 n+1} \circ h_{1}^{-1} \circ h_{2}^{-1} \circ \cdots \circ h_{2 n}^{-1}$ and $h_{2 n-1} \circ h_{2 n-2} \circ \cdots \circ h_{2} \circ h_{1} \circ \alpha_{2 n}$. The f.p. homeomorphism $h$ is defined by

$$
h(x)=\lim _{n \rightarrow \infty} h_{2 n+1} \circ h_{2 n} \circ \cdots \circ h_{1}(x) .
$$

Since we know from the proof that each $x \in N$ is moved only finitely many times by this infinite composition there exists, for any $x \in N$, an $n$ such that $h(x)=h_{2 n+1} \circ h_{2 n} \circ \cdots \circ h_{2} \circ h_{1}(x)$ which is close to $\alpha_{2 n+1}(x)$ (merely!). But $\alpha_{2 n+1}$ is close to $f_{N_{2 n+1}}$ which is given above to be $f F_{1 / m}\left(f^{\prime}\right)^{-1} \pi_{f^{\prime}(N \cup A)}$ letting $m=N_{2 n+1}$.

Therefore $\phi \simeq_{\mathrm{p}} h f^{\prime} \simeq f F_{1 / m}\left(f^{\prime}\right)^{-1} \pi_{f^{\prime}(N \cup A)} f^{\prime}=f F_{1 / m}$ on a certain compact ring of $N$ where choosing the compact ring appropriately closer to $A$ increases $m$. Piecing these homotopies together note that by closeness the latter homotopy above takes the proper map $h f^{\prime}$ to a proper map through proper maps and so is proper.

The final homotopy to check, $f F_{t}$ on pieces, is not hard to recognize as proper. For given a compactum $C^{\prime} \subset N^{\prime}, f^{-1}\left(C^{\prime}\right)=C$ is compact since $f$ is proper. Then for $m$ large $F_{t}, t \in[0,1 / m]$, is the identity on $C$ so inverse images of compacta are compact and $\phi$ is proper fiber homotopic to $f$.

8. Applications. Given a non-compact $Q$-manifold bundle, $p: M \rightarrow$ $B$, the application of the results contained in this paper first requires verification that the bundle is sliced tame at $\infty$ and of sliced finite type as discussed in the introduction. The Homotopy Boundary Theorem then provides a proper fiber homotopy equivalence $f: M \rightarrow N$ to a $Q$-manifold bundle which admits a boundary. The existence of a homeomorphism near $\infty$ in the proper fiber homotopy class of $f$ is then a necessary and sufficient condition for the original bundle to admit a compactification.

In Corollary 3 of the introduction we have assumed sliced tameness at $\infty$ and hence begin by proving the following

Assertion. $p: M \rightarrow S^{1}$ is fiber homotopy equivalent to a compact $Q$-manifold bundle.

Proof. Choose a point $* \in S^{1}$ and let $F=p^{-1}(*)$ be the fiber of the given bundle. The sliced tameness at $\infty$ of $M \rightarrow S^{1}$ restricts to show that 
$F$ is tame at $\infty$. Thus simply choosing a large clean, compact submanifold $F_{1}$ and a homotopy which takes the end $F-F_{1}$ to a compact portion of the space we can feather the homotopy to the identity on $F_{1}-\left(\partial F_{1} \times\right.$ $[0,1)$ ) and observe thus that we can homotope the identity map on $F$ to a push into a finite portion of the space. Triangulation of $Q$-manifolds then implies that $F$ is finitely dominated. Wall's finiteness obstruction assigns to each finitely dominated space $X$ an element $\sigma(X)$ in the reduced projective class group $\tilde{K}_{0}\left(Z \pi_{1} X\right)$ which vanishes if and only if $X$ has finite type. When $X$ is simply connected it is well-known that $\sigma(X)=0$. Thus $F$ is of finite type and we let $f: F \rightarrow P$ be a homotopy equivalence to the compact $Q$-manifold $P$.

Now let the map $\alpha:(I, \partial I) \rightarrow\left(S^{1}, *\right)$ be a wrapping of the unit interval once around $S^{1}$. We obtain a characteristic map (see [11]) $\phi$ : $F \rightarrow F$ of $p: M \rightarrow B$ by lifting $\alpha \circ \Pi_{I}: F \times I \rightarrow S^{1}$ to $\tilde{H}: F \times I \rightarrow M$ with $\tilde{H}_{0}$ the inclusion map and setting $\phi=\tilde{H}_{1}$. Consider the homotopy equivalence $P \stackrel{g}{\rightarrow} F \stackrel{\phi}{\rightarrow} F \stackrel{f}{\rightarrow} P$ where $g$ is a homotopy inverse of $f$. Since $P$ is simply connected the Whitehead group of $P$ is trivial. The map $f \phi g$ is then a simple homotopy equivalence and by the Classification Theorem there exists a homeomorphism $h: P \rightarrow P$ with $h \simeq f \phi g$. Therefore we have a homotopy commutative diagram

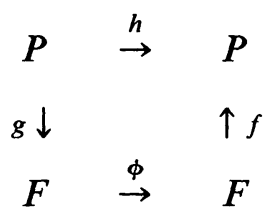

Form the mapping torus of $h, \tau(h)=P \times[0,1] / \sim$ where $\sim$ is the equivalence relationship generated by $(x, 1) \sim(h(x), 0) . \tau(h)$ is a compact $Q$-manifold bundle over $S^{1}$ with characteristic map $h$. Since $h f \simeq f \phi, \tau(h)$ is fiber homotopy equivalent to $p: M \rightarrow S^{1}$ by Lemma 5.2 of [11].

Before we apply our main theorem we calculate $\Pi_{0}$ of the space of concordances of our fiber $F$ and show how this concordance information implies an isotopy result, namely that when $\Pi_{0} \mathscr{C}\left(X^{Q}\right)=0$ proper homotopy classes of homeomorphisms coincide with isotopy classes. The space of concordances of any space $X$, denoted $\mathscr{C}(X)$, is the function space of all homeomorphisms on $X \times I$ which are the identity on $X \times\{0\}$. Chapman in [7] provides exact sequences which relate $\Pi_{0}$ of the space of concordances of a non-compact $Q$-manifold to various limits of $\Pi_{0}$ of the space of concordances of compact submanifolds and complements of compact submanifolds and Whitehead groups of such complements. Using 
these sequences (Theorems 2 and 3 of [7]) and a compact result ( $C$ in [7] which Chapman calculates from [21] and [23]) yields

Proposition 8.1. If $X$ is a simply connected non-compact $Q$-manifold which is simply connected at $\infty$ then $\Pi_{0} \mathscr{C}(X)=0$.

An easy consequence of $\Pi_{0}$ of the concordances of a $Q$-manifold vanishing is the following

Proposition 8.2. Suppose $X$ is a non-compact $Q$-manifold with $\Pi_{0} \mathscr{C}(X)=0$. If $h: X \rightarrow X$ is a homeomorphism which is proper homotopic to the identity then $h$ is isotopic to the identity.

Proof. Replace $X$ by $X \times I$ and let $h: X \times I \rightarrow X \times I$ be a homeomorphism such that $h \simeq_{\mathrm{p}}$ id. In particular then, $\left.h\right|_{X \times 0} \simeq_{\mathrm{p}}(X \times 0 \hookrightarrow X \times$ $I$ ) and since $X \times 0$ is a $Z$-set in $X \times I Z$-set unknotting provides us with an isotopy $H:(X \times I) \times I \rightarrow X \times I$ with $H_{0}=\mathrm{id}_{X \times I}$ and $\left.H_{1} h\right|_{X \times 0}=$ id $_{X \times 0}$. Define $\tilde{H}:(X \times I) \times I \rightarrow X \times I$ by $\tilde{H}=H \circ(h \times$ id $)$ so that $\tilde{H}_{0}=h$ and $\tilde{H}_{1}=H_{1} h$. Since $\left.\tilde{H}_{1}\right|_{X \times 0}=\mathrm{id}_{X \times 0}$ and $\tilde{H}_{t}$ is a homeomorphism for all $t$ we have that $\tilde{H}$ is an isotopy between $h$ and the concordance $\tilde{H}_{1}$.

The assumption that $\Pi_{0} \mathscr{C}(X \times I)=0$ implies that there exists a map $\phi:[1,2] \rightarrow \mathscr{C}(X \times I)$ such that $\phi(1)=\tilde{H}_{1}$ and $\phi(2)=\mathrm{id}_{X \times I}$. The result follows by defining an isotopy $\tilde{\phi}:(X \times I) \times[1,2] \rightarrow X \times I$ from $\tilde{H}_{1}$ to id $X_{X I}$ by setting $\tilde{\phi}_{t}=\phi(t)$.

The following restatement is the form of the above result which we shall use.

Proposition 8.3. Suppose $X$ is a non-compact $Q$-manifold with $\Pi_{0} \mathscr{C}(X)=0$. If $h_{1}, h_{2}: X \rightarrow X$ are homeomorphisms which are proper homotopic then $h_{1}$ is isotopic to $h_{2} . \square$

Returning to the proof of the corollary we apply the Homotopy Boundary Theorem to deduce a proper fiber homotopy equivalence $f$ : $M \rightarrow N$ where $g: N \rightarrow S^{1}$ is a $Q$-manifold bundle which admits a boundary. Splitting both bundles over some point $* \in S^{1}$ yields bundles over the unit interval which are thus products as well as a fiber homotopy equivalence

$$
F \times[0,1] \stackrel{\hat{f}}{\rightarrow} g^{-1}(*) \times[0,1]
$$


Note that we may take $g^{-1}(*)=F$ by Siebenmann's infinite simple homotopy theory [27] and the assumptions on $F$. The identifications $F \times\{0\} \rightarrow F \times\{1\}$ used to recapture the bundles $p: M \rightarrow S^{1}$ and $g:$ $N \rightarrow S^{1}$ from the above products yield homeomorphisms $h_{1}$ and $h_{2}$ from $F$ to itself such that clearly $\tau\left(h_{1}\right) \approx_{\text {f.p. }} M$ and $\tau\left(h_{2}\right) \approx_{\text {f.p. }} N$. But it is easy to see in general that given proper homotopy equivalences $f_{1}: X \rightarrow X$ and $f_{2}: X^{\prime} \rightarrow X^{\prime}$ and a proper fiber homotopy equivalence between $\tau\left(f_{1}\right)$ and $\tau\left(f_{2}\right)$ there exist proper homotopy equivalences $\phi_{1}, \phi_{2}: X \rightarrow X^{\prime}$ such that $\phi_{1} f_{1} \simeq{ }_{\mathrm{p}} f_{2} \phi_{2}$. In our situation observing that the $\phi_{i}$ come from looking at the bundle split over a point of $S^{1}$ and making an appropriate choice of the identification of $g^{-1}(*)$ with $F$ we get $h_{1} \simeq_{\mathrm{p}} h_{2}$. Then Propositions 8.1 and 8.3 yield an isotopy from $h_{1}$ to $h_{2}$, say $G: F \times I \rightarrow F$ with $G_{0}=h_{1}$ and $G_{1}=h_{2}$. Recalling that $\tau\left(h_{i}\right)=F \times[0,1] /(x, 1) \sim\left(h_{i}(x), 0\right)$ define $\tilde{G}: \tau\left(h_{1}\right) \rightarrow \tau\left(h_{2}\right)$ by

$$
\tilde{G}(x, t)=(G(x, 1-t), t) \in \tau\left(h_{2}\right) .
$$

This is a f.p. homeomorphism and so we have shown that $M$ and $N$ are fiberwise homeomorphic and the result is clear.

Corollary 3 and its proof remain valid with any finite one-dimensional polyhedron as base. As a last note we remark that similar calculations involving non-compact $Q$-manifold bundles with slightly more general fibers are possible. For example, results of Bass, Heller and Swann ([1]) and Stallings ([28]) show that $W h(X)=0$ for any space $X$ with $\Pi_{1} X$ free or free abelian. Therefore a non-compact $Q$-manifold bundle over $S^{1}$ which is sliced tame at $\infty$ need only for its fiber to have a free or free abelian fundamental group in order for the proof that it has sliced finite type to go through. Chapman's exact sequences for $\Pi_{0} \mathscr{C}(M)$ do require more care though, e.g. we get $\Pi_{0} \mathscr{C}(M)$ to vanish for $M$ a compact $Q$-manifold (a fact used in the proof of Corollary 3 ) only if $M$ has simply connected components.

\section{REFERENCES}

[1] H. Bass, A. Heller, and R. Swann, The Whitehead group of a polynomial extension, Publ. Math. I.H.E.S. Paris, 22 (1964), 61-79.

[2] K. Borsuk, Theory of Shape, Mathematical Monographs, Vol. 59, Polish Scientific Publishing House, Warsaw. (1975).

[3] W. Browder, J. Levine, and G. R. Livesay, Finding a boundary for an open manifold, Amer. J. of Math., 87 (1965), 1017-1028.

[4] Dan Burghelea, Richard Lashof, and Melvin Rothenberg, Groups of Automorphisms of Manifolds, Springer Lecture Notes, 473 (1975).

[5] T. A. Chapman, On some applications of infinite-dimensional manifolds to the theory of shape, Fund. Math., 76 (1972), 181-193. 
[6] _ Finding boundaries for Hilbert Cube manifolds, preprint.

[7] Concordances of non-compact Hilbert cube manifolds, Pacific J. of Math., 63 (1976), 89-124.

[8] Lectures on Hilbert cube manifolds, C.B.M.S. Regional Conference Series in Math., no. 28, Amer. Math. Soc., 1976.

[9] _ A controlled boundary theorem for Hilbert cube manifolds, Topology Appl., 14 (1982), 247-262.

[10] T. A. Chapman and Steve Ferry, Hurewicz fiber maps with ANR fibers, Topology, 16 (1977), 131-143.

[11] , Fibering Hilbert cube manifolds over ANRs, Compositio Mathematica, Vol. 36, Fasc. 1, (1978), 7-35.

[12] _ The Hauptvermutung for Hilbert cube manifolds and fibered triangulations of Hilbert cube manifold fiber bundles, unpublished manuscript.

[13] , manuscript in preparation.

[14] T. A. Chapman and L. C. Siebenmann, Finding a boundary for a Hilbert cube manifold, Acta. Math., 137 (1976), 171-208.

[15] T. A. Chapman and R. Y. T. Wong, On homeomorphisms of infinite dimensional bundles III, Trans. Amer. Math. Soc., 191 (1974), 269-276.

[16] M. M. Cohen, A Course in Simple-Homotopy Theory, Springer-Verlag, 1973.

[17] A. Dold, Partitions of unity in the theory of fibrations, Ann. of Math., (2) 78 (1963), 223-255.

[18] D. A. Edwards and H. M. Hastings, Every weak proper homotopy equivalence is weakly properly homotopic to a proper homotopy equivalence, Trans. Amer. Math. Soc., 221, No. 1 (1976), 239-248.

[19] Steve Ferry, The homeomorphism group of a compact Hilbert cube manifold is an $A N R$, Ann. of Math., 106 (1977), 101-119.

[20] Ross Geoghegan (ed.), Open problems in infinite-dimensional topology, Topology Proceedings, 4 (1979), 287-338.

[21] A. E. Hatcher, Higher simple homotopy theory I, Ann. of Math., (2) 102 (1975) 101-137.

[22] Concordance Spaces, Higher Simple-Homotopy Theory, and Applications, Proc. of Symp. in Pure Math. Vol. 32 Part I (1978), 3-21.

[23] A. E. Hatcher and J. Wagoner, Pseudo-Isotopies of compact manifolds, Astérisque 6, 1973.

[24] C. Bruce Hughes, Local homotopy properties in spaces of approximate fibrations, Doctoral dissertation, Univ. of Kentucky, 1981.

[25] S. Mardešić and J. Segal, Equivalence of the Borsuk and the ANR-system approach to shapes, Fund. Math., 72 (1971), 61-68.

[26] L. C. Siebenmann, The obstruction to finding a boundary for an open manifold of dimension greater than five, Doctoral dissertation. Princeton Univ., 1965.

[27] , Infinite simple homotopy types, Indag. Math., 32 (1970), 479-495.

[28] J. Stallings, Whitehead torsion of free products, Ann. of Math., 82 (1965), 354-363.

[29] H. Torunczyk and J. West, Fibrations and bundles with Hilbert cube manifold fibers, preprint.

Received September 21, 1983; and in revised form February 12, 1985.

EASTERN KENTUCKY UNIVERSITY

RICHMOND, KY 40475 


\section{PACIFIC JOURNAL OF MATHEMATICS EDITORS}

V. S. VARAdarajan (Managing Editor)
University of California
Los Angeles, CA 90024
CHARLES R. DePrima
California Institute of Technology
Pasadena, CA 91125
R. FINN
Stanford University
Stanford, CA 94305

V. S. VARADARAJAN (Managing Editor)

Hermann FlaschKa

University of Arizona

Tucson, AZ 85721

RAMESH A. GANGOlli

University of Washington

Seattle, WA 98195

ROBION KIRBY

University of California

Berkeley, CA 94720
C. C. MOORE

University of California

Berkeley, CA 94720

H. SAMELSON

Stanford University

Stanford, CA 94305

Harold Stark

University of California, San Diego

La Jolla, CA 92093

\section{ASSOCIATE EDITORS}
R. ARENS
E. F. BECKENBACH
B. H. NeumanN
F. WOLF
K. Yoshida (1906-1982)

\section{SUPPORTING INSTITUTIONS}

\author{
UNIVERSITY OF ARIZONA \\ UNIVERSITY OF BRITISH COLUMBIA \\ CALIFORNIA INSTITUTE OF TECHNOLOGY \\ UNIVERSITY OF CALIFORNIA \\ MONTANA STATE UNIVERSITY \\ UNIVERSITY OF NEVADA, RENO \\ NEW MEXICO STATE UNIVERSITY \\ OREGON STATE UNIVERSITY
}

UNIVERSITY OF OREGON UNIVERSITY OF SOUTHERN CALIFORNIA

STANFORD UNIVERSITY

UNIVERSITY OF HAWAII

UNIVERSITY OF TOKYO

UNIVERSITY OF UTAH

WASHINGTON STATE UNIVERSITY

UNIVERSITY OF WASHINGTON 


\section{Pacific Journal of Mathematics}

\section{Vol. 120, No. $1 \quad$ September, 1985}

Ulrich F. Albrecht, A note on locally $A$-projective groups $\ldots \ldots \ldots \ldots \ldots 1$

Marilyn Breen, A Krasnosel'skiü-type theorem for unions of two starshaped

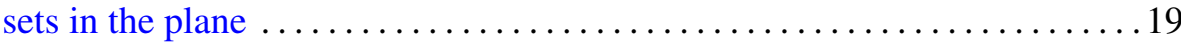

Anthony Carbery, Sun-Yung Alice Chang and John Brady Garnett,

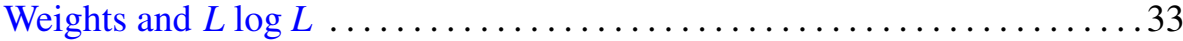

Joanne Marie Dombrowski, Tridiagonal matrix representations of cyclic

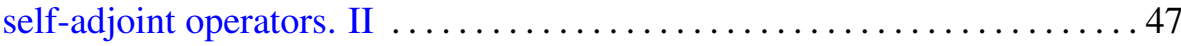

Heinz W. Engl and Werner Römisch, Approximate solutions of nonlinear random operator equations: convergence in distribution $\ldots \ldots \ldots \ldots \ldots 55$

P. Ghez, R. Lima and J. E. Roberts, $W^{*}$-categories $\ldots \ldots \ldots \ldots \ldots \ldots$

Barry E. Johnson, Continuity of homomorphisms of Banach $G$-modules . . 111 Elyahu Katz and Sidney Allen Morris, Free products of topological groups

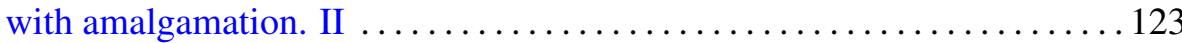

Neal I. Koblitz, $p$-adic integral transforms on compact subgroups of $\mathbf{C}_{p} \ldots 131$ Albert Edward Livingston, A coefficient inequality for functions of positive real part with an application to multivalent functions $\ldots \ldots \ldots 139$

Scott Carroll Metcalf, Finding a boundary for a Hilbert cube manifold

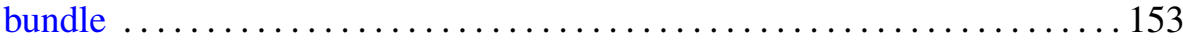

Jack Ray Porter and R. Grant Woods, When all semiregular $H$-closed extensions are compact ........................

Francisco José Ruiz and José Luis Torrea, A unified approach to Carleson measures and $A_{p}$ weights. II $\ldots \ldots \ldots \ldots \ldots \ldots \ldots \ldots \ldots \ldots \ldots \ldots \ldots$

Timothy DuWayne Sauer, The number of equations defining points in general position

John Brendan Sullivan, Universal observability and codimension one subgroups of Borel subgroups

Akihito Uchiyama, Extension of the Hardy-Littlewood-Fefferman-Stein inequality 